\title{
Plasma miR-122 and miR-192 as potential novel biomarkers for the early detection of distant metastasis of gastric cancer
}

\author{
QINGJUAN CHEN $^{1 *}$, XIAOJUN GE ${ }^{2 *}$, YUCHEN ZHANG ${ }^{2}$, HONGWEI XIA $^{2}$, DANDAN YUAN $^{1}$, \\ QIULIN TANG ${ }^{2}$, LIANG CHEN $^{2}$, XIAOHUI PANG $^{1}$, WEIBING LENG $^{1}$ and FENG BI ${ }^{1,2}$ \\ ${ }^{1}$ Department of Medical Oncology, West China Hospital, Sichuan University, Chengdu, Sichuan 610041; \\ ${ }^{2}$ Laboratory of Signal Transduction and Molecular Targeted Therapy, State Key Laboratory of Biotherapy, \\ Sichuan University, Chengdu, Sichuan 610041, P.R. China
}

Received November 15, 2013; Accepted December 30, 2013

DOI: $10.3892 /$ or.2014.3004

\begin{abstract}
The aim of the present study was to ascertain whether plasma levels of specific microRNAs (miRNAs) are associated with distant metastasis (DM) in gastric cancer (GC). miRNA profiling was performed on 12 pairs of samples of gastric cancer with distant metastasis (GC/DM) and gastric cancer with no distant metastasis (GC/NDM); 14 differentially expressed miRNAs were identified for further inspection. Validation of these 14 miRNAs using quantitative reverse transcription PCR (qRT-PCR) on an independent validation set identified 2 differentially expressed miRNAs (miR-122 and miR-192). Further validation of these two candidate miRNAs was conducted in a disease control set, a self-paired plasma set and finally in gastric cell lines in vitro. The results revealed that when compared with GC/NDM and healthy controls (HCs), plasma levels of miR-122 were significantly lower and plasma levels of miR-192 were significantly higher in GC/DM samples (both $\mathrm{P}<0.01$ ). The plasma miR-122 level was again lower and the plasma miR-192-level was again higher in patients with $\mathrm{GC} / \mathrm{DM}$ than in patients with benign gastric ulcer $(\mathrm{BGC})$ and chronic gastritis $(\mathrm{CG})(\mathrm{P}<0.01)$. Compared to the level in patients with pre-distant metastases, miR-122 was significantly decreased while miR-192 was markedly elevated in patients with post-distant metastases $(\mathrm{P}<0.01)$. In CTC105 and CTC141 cells, miR-122 levels were moderately lower and miR-192 levels were markedly higher when compared to the levels in the GES-1 cells. ROC analyses showed that the AUC for plasma miR-122 was 0.808 (95\% CI, 0.712-0.905; $\mathrm{P}<0.01$ ), and the AUC for plasma miR-192 was 0.732 (95\% CI, 0.623-0.841; $\mathrm{P}<0.01$ ) for distinguishing $\mathrm{GC} /$
\end{abstract}

Correspondence to: Professor Feng Bi, Department of Medical Oncology, West China Hospital, Sichuan University, Chengdu, Sichuan 610041, P.R. China

E-mail: bifeng@medmail.com.cn

${ }^{*}$ Contributed equally

Key words: distant metastasis, gastric cancer, miR-122, miR-192, plasma biomarkers
DM from GC/NDM. High expression of miR-122 in plasma independently contributed to a more favorable prognosis for GC (hazard ratio, 0.262; 95\% CI, 0.164-0.816; $\mathrm{P}=0.038$; Cox regression analysis), whereas the miR-192 level was not associated with the overall survival time. Our results demonstrated that assessment of decreased circulating miR-122 and elevated circulating miR-192 levels has the potential to improve early detection of DM in GC. Higher plasma levels of miR-122 in $\mathrm{GC}$ may indicate a favorable prognosis.

\section{Introduction}

Although in recent years a downward trend in the global incidence of gastric cancer (GC) has been noted, GC still ranks worldwide as the fourth most common cancer and is the second leading cause of cancer-related mortality (1-3). In China, the annual prevalence and death rates for GC are more than twice the world average, with mortality from GC accounting for almost a quarter (23.2\%) of cancer-related deaths (4).

Almost one-third of GC cases present with metastasis at the time of diagnosis, and 4-14\% have metastatic disease to the liver $(3,5,6)$. Gastric cancer with distant metastasis (GC/DM) has a poor prognosis, with a median survival of only 3-5 months (7-9). Systemic chemotherapy is the preferred treatment for metastatic disease and may extend overall survival to $8-12$ months $(10,11)$. The efficacy of surgery is controversial, and only some rigorously selected cases may benefit from surgery. Palliative surgery in these patients is not recommended according to treatment guidelines $(12,13)$. Therefore, effective diagnostic markers are needed to allow the early diagnosis of GC/DM. At present, detection of distant metastases mainly depends on computed tomography (CT) investigations, but it can be difficult to identify tiny or occult lesions. Thus, this technique is of limited diagnostic value. Consequently, assessment of a large number of recently identified blood tumor markers, such as circulating microRNAs (miRNAs) has become a central focus of research.

miRNAs are a class of non-coding small RNAs, usually 19-25 nucleotides long, which are transcribed from a stem-loop structure precursor (14). miRNAs mainly regulate target gene mRNAs by degradation or inhibition of translation through complete or incomplete matching of 3'UTR of the target gene, 
and, thus, participate in signaling pathways affecting tumor occurrence and development, playing similar roles to those of oncogenes or tumor-suppressor genes, in either promoting or inhibiting tumor development $(15,16)$. Differentially expressed miRNAs have been found in many types of tumors $(17,18)$, and different types of cancers exhibit distinct miRNA profiles $(15,19)$. Moreover, many studies have shown that miRNAs are stable in blood samples, thus, making the quantitative detection of miRNAs in blood feasible. Therefore, miRNAs in blood have been extensively studied as biomarkers for GC diagnosis, prognosis and treatment (20-23), yet, specific circulating miRNAs associated with distant metastasis in GC remain unidentified.

The aim of the present study was to identify specific miRNAs in GC/DM plasma samples. Using miRNA microarray screening and subsequent qRT-PCR validation, we identified 14 miRNAs in the plasma of patients with GC/DM. Of these miRNAs, we focused on two, miR-122 and miR-192, and found that compared with gastric cancer with no distant metastasis (GC/NDM), miR-122 expression was significantly lower, whereas miR-192 expression was significantly higher in GC/DM. In addition, the potential use of these miRNAs as markers for GC/DM was assessed in one gastric mucosal cell line, three human GC cell lines, and two gastric circulating tumor cell lines. Finally, survival analysis was performed to clarify whether the specific miRNA expression level was associated with patient survival time.

\section{Materials and methods}

Patient samples. The study was approved by the Clinical Research Ethics Committee of West China Hospital, and all participants provided informed consent.

In total, 96 patients with GC, 7 patients with BGC, 10 patients with $\mathrm{CG}$ and 36 control subjects were enrolled in the study. The patients with GC consisted of 48 cases with GC/DM and 48 cases with GC/NDM. Plasma samples were obtained from the patients at the Department of Abdominal Cancer, West China Hospital of Sichuan University, during the period March 2012 to April 2013. The 48 GC/DM cases included GCs with liver, lung, or bone metastasis, or simultaneous multiple-organ metastasis, supported by imaging and pathological evidence. The $48 \mathrm{GC}$ cases without DM were at a local-advanced stage, accompanied by a giant lump or distant lymph node (such as supraclavicular lymph node) metastasis, but had no pathological evidence of DM. Patients in the disease control set had no pathological evidence of tumor disease and were matched to patients by age and gender.

The control plasma samples were obtained from individual undergoing a routine physical examination, who showed no evidence of disease. Controls (HCs) were matched to patients by age and gender.

Study design. The study used a six-step procedure. In step 1, 12 pairs of GC/DM and GC/NDM samples (the discovery set) were set up for miRNA microarray screening. The miRNA profiles were then generated, and 14 miRNAs that were found to be differentially expressed were selected for validation.

In step 2, quantitative reverse transcription-PCR (qRT-PCR) was used to investigate for the presence of the 14 selected miRNAs in the validation set, which consisted of 72 plasma samples from GC (36 pairs of GC/DM and GC/NDM samples) and 36 plasma samples from HCs. This step identified 2 miRNAs (miR-122 and miR-192) that were differentially expressed.

In step 3, to ascertain whether the two identified miRNAs (miR-122 and miR-192) are specific to GC/DM, qRT-PCR validation was further conducted on a cohort containing disease controls, consisting of 10 patients with $\mathrm{CG}, 7$ patients with benign $\mathrm{BGC}$, as well as $10 \mathrm{GC} / \mathrm{DMs}$ and $10 \mathrm{HC}$ samples.

In step 4, the levels of the selected miRNAs (miR-122 and miR-192) were also examined using paired pre- and postdistant metastasis plasma samples from 7 patients.

In step 5, final validation was conducted using gastric cell lines: a normal gastric mucosal cell line (GES-1), GC cell lines (SGC7901, MKN45 and BGC823), and cell lines established from circulating tumor cells (CTC141 and CTC105).

In step 6, we evaluated the diagnostic value of these markers for predicting DM. Using Youden's index as the criterion for determining the optimal cut-off value, the area under the curve (AUC) of each receiver operating characteristic (ROC) curve was calculated, and survival analysis was also conducted for these two miRNAs.

Gastric cancer cell lines. The human gastric mucosal cell line GES-1, and the human GC cell lines SGC7901, MKN45 and BGC823 were maintained in our laboratory. The circulating tumor cell lines CTC141 and CTC105 were a kind gift from the Laboratory of Stem Cell Biology of Sichuan University. These two cell lines are CD44-positive cells with great potential for tumor metastasis, and were derived from blood samples of two patients with gastric adenocarcinoma by magnetic isolation, purification and stable passaging (24).

All six cell lines were cultured in RPMI-1640 medium supplemented with $10 \%$ fetal bovine serum (FBS) in $5 \% \mathrm{CO}_{2}$ at $37^{\circ} \mathrm{C}$.

Plasma preparation and RNA isolation. All plasma samples were collected prior to any therapeutic procedure such as surgery, chemotherapy or radiotherapy. Venous blood (3-5 ml) was collected into tubes containing EDTA, and the separation procedure was carried out within $2 \mathrm{~h}$ after venipuncture. The samples were centrifuged at $1900 \mathrm{x}$ g for $10 \mathrm{~min}$ at $4^{\circ} \mathrm{C}$, followed by a high-speed centrifugation step of $13,000 \times \mathrm{g}$ for a further $10 \mathrm{~min}$ at $4^{\circ} \mathrm{C}$ to complete the removal of residual cell debris and genomic DNA. The supernatant plasma was then removed, split into aliquots and frozen at $-80^{\circ} \mathrm{C}$ until use.

Total RNA was extracted from plasma using TRIzol LS reagent (Invitrogen, Carlsbad, CA, USA) and from cells using TRIzol reagent (Invitrogen). After adding $750 \mu 1$ TRIzol LS to $250 \mu \mathrm{l}$ plasma, 1 pmol ath-miR-159a (synthesized by Riobio, Guangzhou, China) was added as spike-in control and the solution was mixed thoroughly. Total RNA was extracted by TRIzol LS, following the manufacturer's protocol with minor modifications. The RNA pellet was resuspended in $25 \mu \mathrm{l}$ nuclease-free water and stored at $-80^{\circ} \mathrm{C}$.

miRNA profiling. Using 1 pooled plasma sample from 12 GC/DM samples and 1 pooled plasma sample from $12 \mathrm{GC} /$ NDM samples, miRNA profiling was performed with miRCURY LNA ${ }^{\mathrm{TM}}$ Universal RT microRNA PCR assays 
(Exiqon, Vedbaek, Denmark). The assays used the SerumPlasma Focus microRNA PCR Panels (Exiqon) comprising a 168 miRNA primer set for disease-associated miRNAs commonly found in human serum/plasma, along with $\mathrm{LNA}^{\mathrm{TM}}$ enhanced primers tagged with SYBR-Green. The method used 384-well PCR plates, and the pre-aliquoted LNA ${ }^{\mathrm{TM}}$ PCR primers were added to each well. Total RNA was prepared as described above, and an amount of 22 ng of RNA was used for profiling in each reaction. cDNA was pre-amplified by primers SP6, after identifying Ct reunification values, then doing follow-up experiments. In total, 168 target miRNAs were detected, and the fold-change in the expression of each miRNA was calculated.

qRT-PCR validation of miRNAs in plasma. The miRNA profiling identified 14 differentially expressed miRNAs, which were selected and further quantified by qRT-PCR. Specific primers were purchased from Tiangen Biotechnology (Beijing, China). An aliquot (3 $\mu \mathrm{l})$ of total RNA was obtained from the $25 \mu \mathrm{l}$ solution of the resuspended total RNA and was polyadenylated and reverse-transcribed to cDNA using a commercial kit (One Step PrimeScript miRNA cDNA Synthesis kit; Takara, Tokyo, Japan). Subsequently, the cDNA was diluted 7.5-fold, and $2 \mu \mathrm{l}$ of this diluted cDNA was used as a template. Finally, qRT-PCR was run on a PCR system (IQ5; Bio-Rad Laboratories, Inc., Hercules, CA, USA) with miRNA-specific primers and SYBR-Green Mix (Takara) according to the manufacturer's instructions. The cycle threshold $(\mathrm{Ct})$ values of miRNA expression were normalized to ath-miR-159a (U6).

Further validation of miRNAs in cell lines by qRT-PCR. Using the process described above, two miRNAs (miR-122 and miR-192) were selected for further validation in GES-1, a normal gastric mucosal cell line; two cell lines with low differentiation potential, BGC823 and MKN45; SGC7901, a cell line with moderate differentiation; and the blood circulating tumor cell lines CTC141 and CTC105. qRT-PCR was carried out as described above, except that U6 was used as an internal control in the cells (thus no spike-in control was needed) and the cDNA was diluted 40-fold.

Statistical analysis. The expression levels of miRNAs are presented as $2^{-\Delta \mathrm{Ct}}$, where the $\Delta \mathrm{Ct}$ sample is defined as $\mathrm{Ct}_{(\text {target })^{-}}$ $\mathrm{Ct}_{(\text {miR-159) }}$, and $\mathrm{Ct}$ represents the threshold cycle number, shown as median $\pm \mathrm{SD}$. The $2^{-\Delta \Delta \mathrm{Ct}}$ method was used to analyze the relative expression of miRNAs, and the Mann-Whitney or Kruskal-Wallis test to compare the expression of plasma miRNAs between the different groups. ROC curves and AUC were used to evaluate the feasibility of using specific plasma miRNAs as a diagnostic tool for GC/DM. Probability of survival was determined by Kaplan-Meier analysis, and the significance of differences between groups was analyzed by the log-rank test. The fold-change (compared with paired samples in the HC group) was analyzed using $>2$ or $<2$ as the miRNA expression cut-off value. Cox proportional-hazards regression analysis was applied to estimate hazard ratios for survival. The relationship between clinicopathologic features was assessed by ANOVA, Student's t-test or $\chi^{2}$ test. All tests were two-sided, and a significance level of $\mathrm{P}<0.05$ was consid- ered to indicate a statistically significant result. Statistical analysis was performed using SPSS software (version 16.0; SPSS Ltd., Woking, Surrey UK). Graphs and charts were created using the software packages GraphPad Prism (version 5.00 for Windows; GraphPad Software Inc., La Jolla, CA, USA) and MedCalc (version 12.3.0.0; MedCalc Software, Ostend, Belgium).

\section{Results}

Patients and groups. The baseline epidemiological and clinical characteristics of the 113 patients and the $36 \mathrm{HCs}$ enrolled in the present study are shown in Table I.

There were no significant differences in the distribution of age or gender in the discovery set, the validation set and the disease control set. The pathological differentiation, TNM stage, and tumor location did not differ between the GC/DM and GC/NDM groups, and the distribution of metastatic location in the GC/DM group also did not differ between the discovery set and the validation set.

miRNA profiling analysis. RNA quantification and quality assurance of total RNA extracted from the two pooled samples (equal amount from 12 pairs of GC/DM and GC/NDM) showed that the concentration was 11.94 and $11.31 \mathrm{ng} / \mu \mathrm{l}$, respectively. The miRNA profiling identified 14 significantly altered miRNAs (miR-122, miR-125a-5p, miR-126, miR-133b, miR-148b, miR-151-3p, miR-15b, miR-192,miR-195, miR-200c, miR-205, miR-320a, miR-346 and miR-136) (Table II).

Validation of the plasma-specific miRNAs for the three groups in the validation set. These 14 miRNAs were then assessed to identify those with a mean $\mathrm{Cq}$ value of $<35$, fold-change $>2$ and $\mathrm{P}<0.05$. Using this method, we found 2 of the 14 miRNAs (namely, miR-122 and miR-192) to have significant expression in the plasma samples, and these were selected for further analysis.

Expression of miR-122 was significantly lower, and expression of miR-192 was significantly higher (both $\mathrm{P}<0.01$ ) in the GC/DM when compared to levels in the GC/NDM and HC groups, and there were no significant differences in the levels of miR-122 or miR-192 between the GC/NDM and HC groups ( $\mathrm{P}>0.05$ ) (Fig. 1A and B). Using the AUC of the ROC curve to estimate the diagnostic value of miR-122 or miR-192 in discerning distant metastasis in GCs, we found that the plasma levels of miR-122 and miR-192 effectively distinguished patients with GC/DM from patients with GC/NDM and from the HCs (Fig. 1C-F).

Detection of candidate miRNAs in the disease control set. To ascertain whether the upregulated expression of miR-122 and miR-192 was specific to GC/DM, we evaluated the miR-122 and miR-192 expression in plasma from the controls with two additional diseases: 7 patients with BGU, 10 with CG. miR-122 and miR-192 expression in these diseases was compared with that of GC/DM and the controls. Results showed that miR-122 was lower and miR-192 was higher in patients with $\mathrm{DM}(\mathrm{P}<0.01)$ than in the patients with the other two diseases (Fig. 2A and B), confirming that lower miR-122 and higher miR-192 levels are typical of GC/DM. 
Table I. Clinicopathological features of the GC cancer with distant metastasis and without distant metastasis and the healthy controls.

\begin{tabular}{|c|c|c|c|c|c|c|c|}
\hline \multirow[b]{3}{*}{ Variables } & \multicolumn{7}{|c|}{ Number of cases $(\%)$} \\
\hline & \multicolumn{2}{|c|}{$\begin{array}{c}\text { Discovery set } \\
\text { (pooled samples) }\end{array}$} & \multicolumn{3}{|c|}{$\begin{array}{c}\text { Validation set } \\
\text { (independent samples) }\end{array}$} & \multicolumn{2}{|c|}{$\begin{array}{c}\text { Disease } \\
\text { control set }\end{array}$} \\
\hline & $\begin{array}{l}\text { GC/DM } \\
(n=12)\end{array}$ & $\begin{array}{c}\text { GC/NDM } \\
(n=12)\end{array}$ & $\begin{array}{c}\text { GC/DM } \\
(n=36)\end{array}$ & $\begin{array}{c}\text { GC/NDM } \\
(\mathrm{n}=36)\end{array}$ & $\begin{array}{c}\mathrm{HC} \\
(\mathrm{n}=36)\end{array}$ & $\begin{array}{l}\text { BGC } \\
(n=7)\end{array}$ & $\begin{array}{c}C G \\
(n=10)\end{array}$ \\
\hline \multicolumn{8}{|l|}{ Age (years) } \\
\hline Median & 57 & 58 & 56 & 57 & 59 & 58 & 57 \\
\hline Range & $44-62$ & $45-60$ & $48-61$ & $51-66$ & $48-61$ & $54-60$ & $52-61$ \\
\hline \multicolumn{8}{|l|}{ Gender } \\
\hline Male & $9(75.0)$ & $9(75.0)$ & $28(77.8)$ & $26(72.2)$ & $28(77.8)$ & $5(71.4)$ & $7(70.0)$ \\
\hline Female & $3(25.0)$ & $3(25.0)$ & $8(22.2)$ & $10(27.8)$ & $8(22.2)$ & $2(28.6)$ & $3(30.0)$ \\
\hline \multicolumn{8}{|l|}{ TNM stage } \\
\hline III & $4(33.3)$ & $\begin{array}{ll}0 & (0.0)\end{array}$ & $13(36.1)$ & $0 \quad(0.0)$ & & & \\
\hline IV & $8(66.7)$ & $12(100.0)$ & $23(63.9)$ & $36(100.0)$ & & & \\
\hline \multicolumn{8}{|l|}{ Pathology } \\
\hline Well & $1(8.3)$ & $2(16.7)$ & $3(8.3)$ & $4(11.1)$ & & & \\
\hline Moderate and poor & $11(91.7)$ & $10(83.3)$ & $33(91.7)$ & $32(88.9)$ & & & \\
\hline \multicolumn{8}{|l|}{ Tumor location } \\
\hline Cardia & $4(33.3)$ & $4(33.3)$ & $14(38.9)$ & $19(52.8)$ & & & \\
\hline Body & $4(33.3)$ & $3(25.0)$ & $7(19.4)$ & $3(8.3)$ & & & \\
\hline Antrum & $4(33.3)$ & $5(41.7)$ & $15(41.7)$ & $14(38.9)$ & & & \\
\hline \multicolumn{8}{|l|}{ Metastasis location } \\
\hline Liver & $6(50.0)$ & & $22(61.1)$ & & & & \\
\hline Lung & $3(25.0)$ & & $9(25.0)$ & & & & \\
\hline Bone & $2(16.7)$ & & $2(5.6)$ & & & & \\
\hline Liver + lung & $1(8.3)$ & & $3(8.3)$ & & & & \\
\hline
\end{tabular}

GC, gastric cancer; HC, healthy controls; GC/DM, GC with distant metastasis; GC/NDM, GC with no distant metastasis; BGC, benign gastric ulcer; CG, chronic gastritis.

Table II. Dysregulated miRNAs in the discovery set found through miRCURY LNA ${ }^{\mathrm{TM}}$ profiling.

\begin{tabular}{|c|c|c|c|c|c|}
\hline \multirow[b]{2}{*}{ miRNA ID } & \multicolumn{2}{|c|}{ Mean $\Delta \mathrm{Ct}\left(\mathrm{Ct}_{\mathrm{Tar}}-\right.$ mean $\left.\mathrm{Ct}_{\mathrm{Ref}}\right)$} & \multicolumn{2}{|c|}{$2^{-\Delta \mathrm{Ct}}$} & \multirow[b]{2}{*}{ Fold difference (DM/NDM) } \\
\hline & GC/DM & GC/NDM & GC/DM & GC/NDM & \\
\hline $\operatorname{miR}-122$ & 4.194 & -3.348 & 0.055 & 10.184 & 0.005 \\
\hline $\operatorname{miR}-125 a-5 p$ & 1.339 & -1.431 & 0.395 & 2.697 & 0.147 \\
\hline miR-126 & -1.547 & -3.651 & 2.921 & 12.566 & 0.232 \\
\hline $\operatorname{miR}-133 b$ & 4.194 & -0.357 & 0.055 & 1.281 & 0.043 \\
\hline $\operatorname{miR}-148 b$ & 4.194 & 0.489 & 0.055 & 0.712 & 0.077 \\
\hline $\operatorname{miR}-151-3 p$ & 2.988 & 1.086 & 0.126 & 0.471 & 0.268 \\
\hline $\operatorname{miR}-15 b$ & 1.635 & -1.503 & 0.322 & 2.834 & 0.114 \\
\hline miR-192 & -1.617 & 3.771 & 3.068 & 0.073 & 41.883 \\
\hline miR-195 & 4.194 & 0.415 & 0.055 & 0.75 & 0.073 \\
\hline miR-200c & 4.194 & -1.071 & 0.055 & 2.101 & 0.026 \\
\hline miR-205 & 4.194 & -0.324 & 0.055 & 1.252 & 0.044 \\
\hline miR-320a & -1.138 & -4.296 & 2.2 & 19.639 & 0.112 \\
\hline miR-346 & -2.502 & 3.771 & 5.666 & 0.073 & 77.356 \\
\hline miR-136 & 1.451 & 3.771 & 0.366 & 0.073 & 4.994 \\
\hline
\end{tabular}

GC, gastric cancer; GC/DM, GC with distant metastasis; GC/NDM, GC with no distant metastasis. 
A

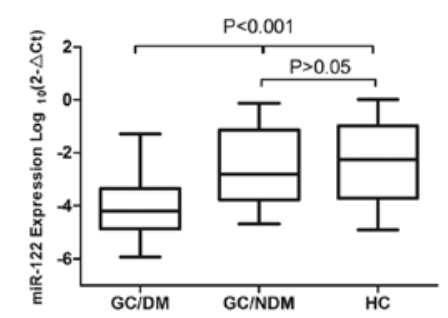

C

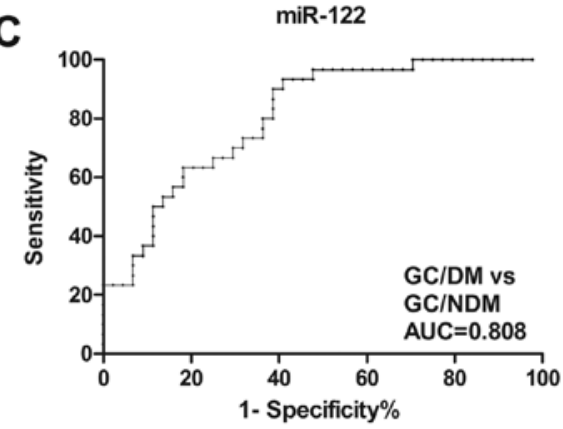

E

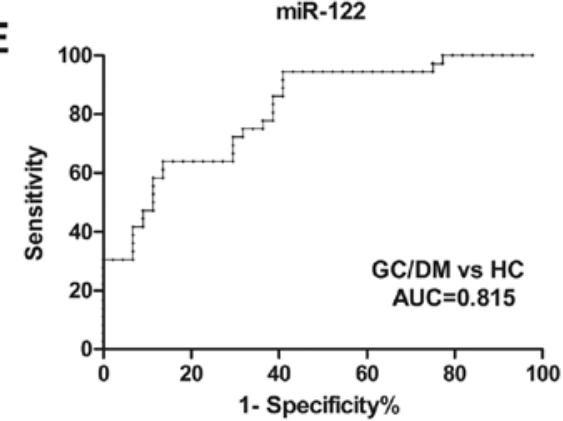

B

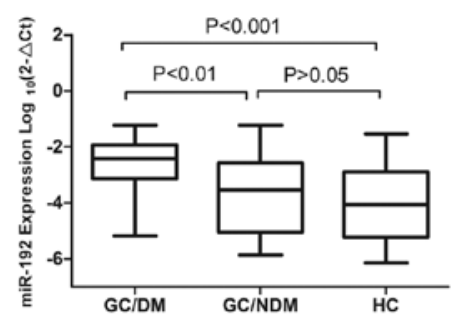

D
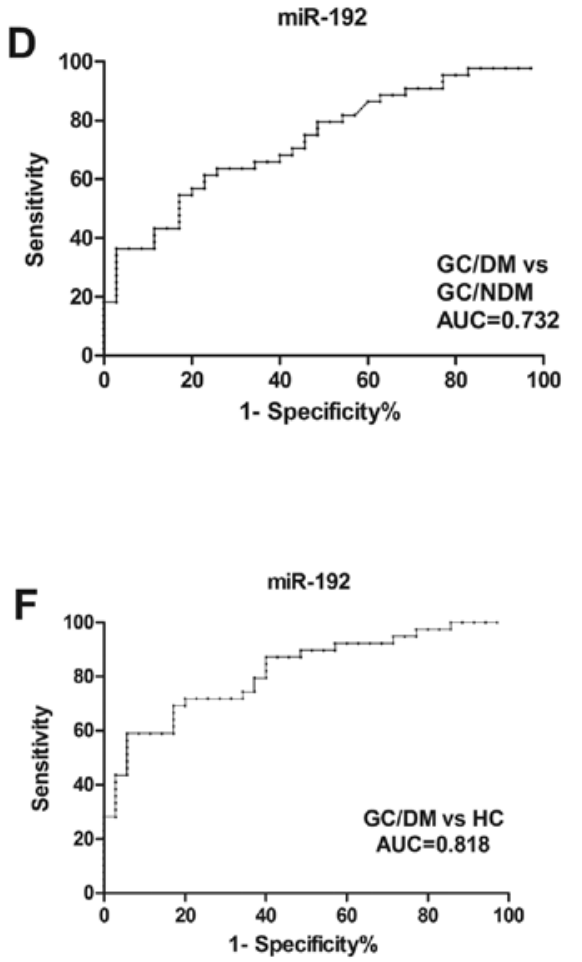

Figure 1. (A and B) Box plots of plasma levels $\left(2^{-\Delta C}\right)$ of miR-122 and miR-192 in the GC/DM ( $\left.n=36\right)$, GC/NDM ( $\left.n=36\right)$ and HC ( $\left.=36\right)$ groups. Expression levels of the miRNAs ( $\log 10$ scale on the y-axis) were normalized to ath-miR-159a. The line represents the median value. (A) Expression of miR-122 in plasma samples was significantly reduced in the GC/DM group when compared with the expression in the GC/NDM and HC groups. (B) Expression of miRNA-192 was significantly elevated in the GC/DM group when compared with expression in the GC/NDM and HC groups. (C and F) Diagnostic efficiency of miR-122 and miR-192 in the three groups. The AUC of the ROC curve for detecting DM from NDM was 0.808 for miR-122 and 0.732 for miR-192 (both P<0.001). The AUC of the ROC curve for detecting DM from HC was 0.815 for miR-122 and 0.818 for miR-192 (both P<0.001). GC, gastric cancer; GC/DM, GC with distant metastasis; GC/NDM, GC with no distant metastasis; HC, healthy controls; AUC, area under the curve; ROC, receiver operating characteristic.

Validation of the candidate miRNAs in self-paired plasma samples in pre- and post-distant metastasis plasma samples. By comparison of the samples of patients before distant metastases, we found that miR-122 was significantly decreased while miR-192 was dramatically elevated in patients after distant metastases ( $\mathrm{P}<0.01$; Fig. $2 \mathrm{C}$ and $\mathrm{D})$.

Further validation using gastric cell lines. Compared with the GES-1 cells, CTC105 and CTC141 cells showed miR-122 levels that were 1.9- and 2.1-fold lower, respectively, and miR-192 levels that were 8.5- and 11.0-fold higher. The expression of both miRNAs in the other three cell lines varied (Fig. 3A and B).

Kaplan-Meier survival analysis. Finally, we determined whether the expression levels of miR-122 and miR-192 are correlated with the survival of patients with GC. Using the Kaplan-Meier and log-rank methods, we found that high expres- sion of plasma miR-122 was correlated with prolonged overall survival (OS) of patients with GC. Univariate Cox analysis revealed that TNM stage (IV) and differentiation (poor) were significantly associated with reduced patient survival, while the plasma miRNA level (high miR-122) contributed to better prognosis of patients with GC. Multivariate Cox analysis indicated that upregulation of plasma miR-122 independently contributed to a more favorable prognosis of patients with GC (hazard ratio, 0.262; 95\% CI, 0.164-0.816; $\mathrm{P}=0.038$ ), while the level of miR-192 was not associated with OS in patients with GC (Table III and Fig. 4A and B).

\section{Discussion}

In the present study, we used a case-control experiment to investigate the role of plasma miRNAs in predicting GC/DM using miRNA profiling on two pooled samples, followed by qRT-PCR assays on an independent validation set and then 
A

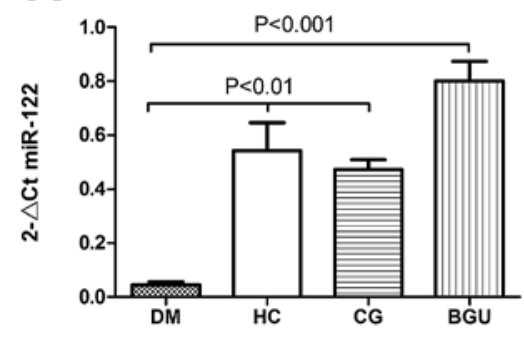

C

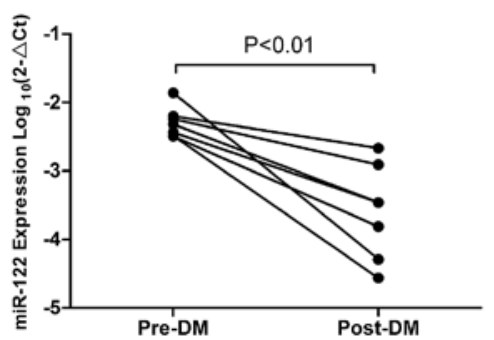

B

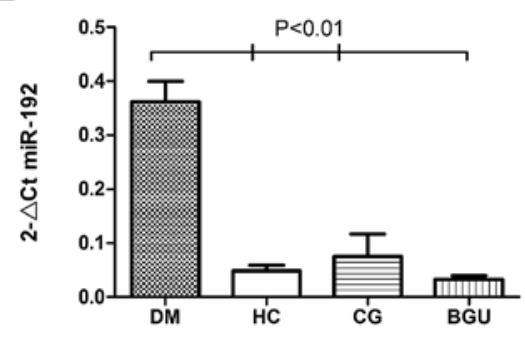

D

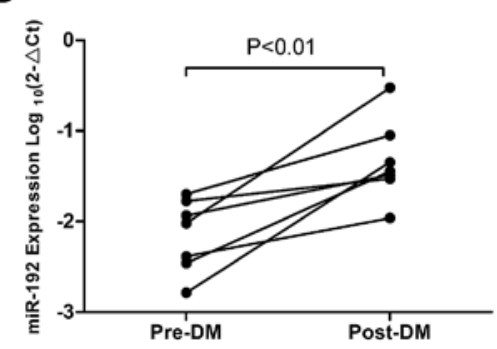

Figure 2. (A and B) miR-122-5p and miR-192 expression levels were evaluated by real-time PCR in plasma from $10 \mathrm{GC} / \mathrm{DM}$ patients, $10 \mathrm{HCs}, 10 \mathrm{chronic}$ gastritis (CGs) and 7 benign gastric ulcer (BGC) patients. (A) The miR-122-5p level was significantly lower in patients with GC/DM than in patients with BGCs and in the HCs. (B) The miR-192 level was significantly higher in patients with GC/DM than in those with BGC and in the HCs (P<0.01). (C and D) Changes in plasma levels of (C) miR-122 and (D) miR-192 in patients with gastric cancer ( $\mathrm{n}=7)$ before (Pre-DM) and after distant metastasis $($ Post-DM) $(\mathrm{P}<0.01)$.

A

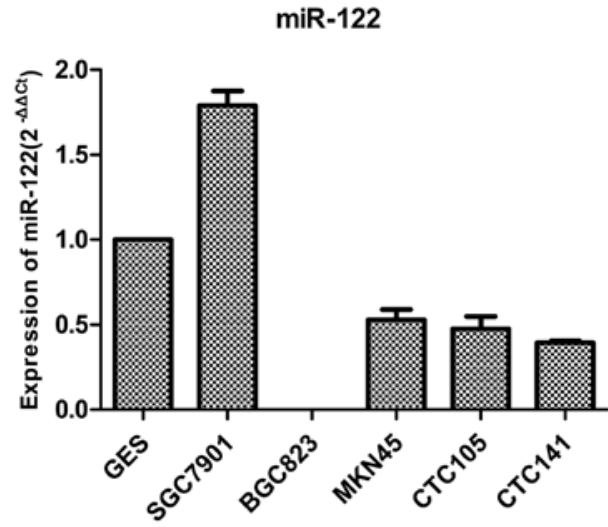

B

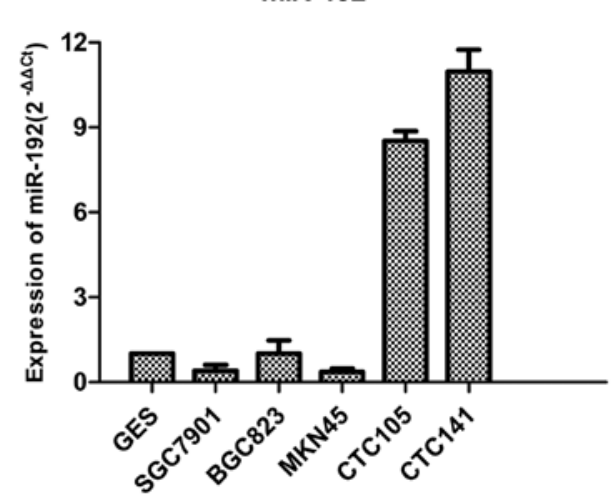

Figure 3. Expression of miR-122 and miR-192 was detected by qRT-PCR in the normal gastric mucosal cell line GES-1; two cell lines with low differentiation potential, BGC823 and MKN45; a cell line with moderate differentiation, SGC7901; and the blood circulating tumor cell lines CTC141 and CTC105. (A) Expression of miR-122 in the SGC7901 cell line was 1.8-fold higher than that in the GES-1 cell line, while in MKN45, CTC105 and CTC141 cells it was 1.9-, 2.1- and 2.5-fold lower than that in the GES-1 cell line. Expression of miR-122 in the BGC823 cell line was almost absent when compared to the expression in the GES-1 cell line. (B) Compared with expression in the GES-1 cell line, expression of miR-192 in SGC7901 and MKN45 cells was $2.5-$ and 2.8-fold lower, respectively; expression of miR-192 in the CTC105 and CTC141 cells was 8.5- and 11.0-fold higher, respectively; and expression of miR-192 in the BGC823 cells was approximately the same.

later uisng gastric cancer cell lines. Plasma obtained from 12 cases of each of the two patient groups in the discovery set were mixed together to form two pooled plasma samples that were used for subsequent experiments. This method of pooling samples is a widely used technique that has been shown to be reliable for profiling (25-28).

Tumor metastasis involves a number of critical steps, including epithelial-mesenchymal transition (EMT), apoptosis and angiogenesis. Multiple miRNAs have been shown to promote or inhibit metastasis (29). Previous studies have shown that miR-200c is closely associated with cancer metastasis and acts as a metastamiR in the EMT process (30). By contrast, miR-31 inhibits multiple steps of metastasis, including invasion, anoikis and colonization, and was shown to cause a $95 \%$ reduction in lung metastasis in a model of breast cancer (31).
In the present study, using miRNA profiling screening, we identified a total of 14 differentially expressed miRNAs (see Material and methods). These miRNAs were then selected and validated, and two miRNAs, miR-122 and miR-192, were selected for further investigation. The plasma levels of these miRNAs, but not of the other tested miRNAs, were significantly different between the GC/DM and GC/NDM patients in this population.

miR-122 was initially discovered as a liver-specific miRNA, and its precursors are found on human chromosome 18 , at position $18 \mathrm{q} 21(32,33)$. miR-122 is physiologically involved in liver cell metabolism, cell differentiation and other emergency responses, while in pathological states, it promotes hepatitis $C$ virus replication in liver cells, and participates in carcinogenesis, development and metastasis 
Table III. Univariate and multivariate analyses of plasma miR-122 and clinicopathological factors associated with survival in gastric cancer.

\begin{tabular}{|c|c|c|c|c|c|c|}
\hline \multirow[b]{2}{*}{ Variable } & \multicolumn{3}{|c|}{ Univariate analysis } & \multicolumn{3}{|c|}{ Multivariate analysis } \\
\hline & Hazard ratio & $95 \%$ CI & P-value & Hazard ratio & $95 \% \mathrm{CI}$ & P-value \\
\hline Differentiation (poor) & 3.162 & $1.065-8.716$ & 0.037 & 2.215 & $0.840-5.839$ & 0.108 \\
\hline TNM stage (IV) & 2.216 & $1.209-4.587$ & 0.027 & 2.963 & $1.072-8.191$ & 0.036 \\
\hline Metastasis location (lung) & 2.228 & $0.954-5.202$ & 0.064 & $(-)$ & $(-)$ & $(-)$ \\
\hline Plasma miR-192 level (low) & 1.049 & $0.403-1.936$ & 0.921 & 1.119 & $0.490-2.556$ & 0.789 \\
\hline Plasma miR-122 level (high) & 0.237 & $0.021-0.896$ & 0.045 & 0.262 & $0.164-0.816$ & 0.038 \\
\hline
\end{tabular}
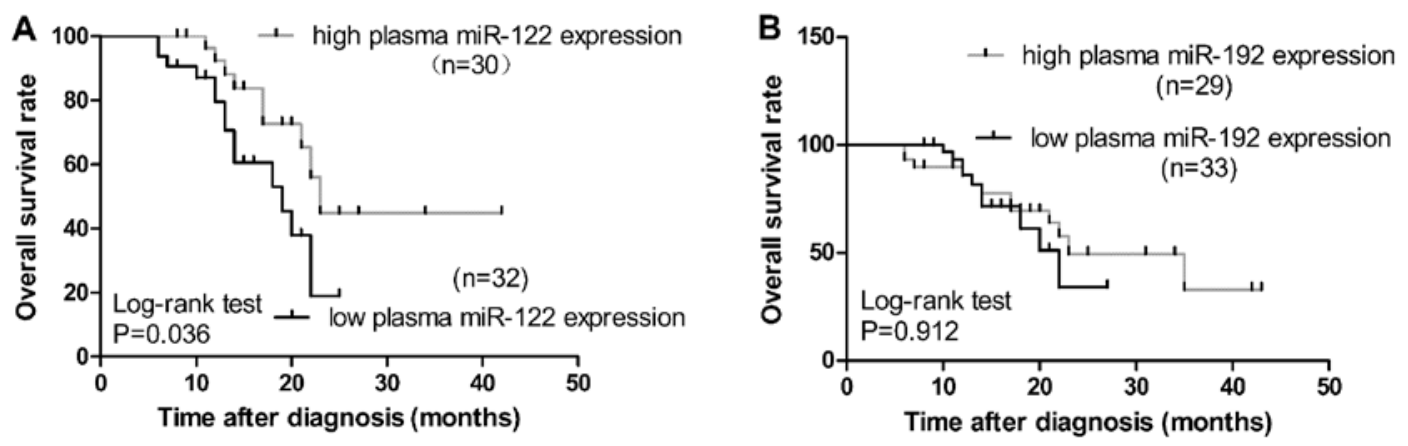

Figure 4. Kaplan-Meier survival curves for patients with GC in the validation set plotted for plasma miR-122 and miR-192 levels. The P-value was calculated using the log-rank test between patients with high fold-change $(>2)$ and low fold-change $(<2)$. (A) The OS rate of patients with GC with high plasma miR-122 expression was significantly higher than the OS in the patients with low plasma miR-122 expression $(\mathrm{P}=0.036)$. (B) Survival analysis showed that the expression level of miR-192 had no relationship with OS (P=0.912). GC, gastric cancer; OS, overall survival.

of hepatocellular carcinoma (34). A recent study found that higher expression of circulating miR-122 predicts metastasis in early-stage breast cancer (35). Iino et al (36) found that miR-122 was significantly upregulated in synchronous colorectal liver metastasis, and also negatively regulated its target gene, cationic amino acid transporter 1 . In contrast, in the present study, we found significantly reduced levels of plasma miR-122 in patients with GC/DM relative to patients with GC/NDM and to the HCs, in accordance with another previous study that showed that miR-122 is downregulated in cancer $(37,38)$.

Previous studies found that miR-192 is significantly increased in many types of cancer (39-41), but decreased in many others $(42,43)$. Studies have also shown that p53-mediated upregulation of miR-192 prevents EMT in hepatocellular carcinoma through repression of the transcription factor ZEB2 (44). In the present study, we found that miR-192a was present at significantly higher levels in patients with GC/DM, compared with patients with GC/NDM, the HCs as well as the patients with gastric benign lesions, which is consistent with previous results. This suggests that miR-192 is involved in DM from GC, and might be a suitable marker for discriminating GC/DM from GC/NDM.

We also tested miR-122 and miR-192 in the GC cell lines CTC105 and CTC141, and found that, compared with levels in the GES-1 cells, miR-122 levels were moderately lower while miR-192 levels were dramatically higher. Since CTC105 and CTC141 cells were established from circulating tumor cells possessing metastatic potential, the expression of
miR-122 was lower in these two original metastatic cell lines than in the GES-1 cell line, while miR-192 showed the opposite trend. Compared with levels in the GES-1 cells, miR-122 was moderately increased in the SGC7901 cells but slightly decreased in the MKN45 cells and was almost absent in the BGC823 cells. By contrast, miR-192 levels in the SGC7901 and MKN45 cells were considerably lower than that in the GES-1 cells, but levels in the BGC823 cells were almost the same as in the GES-1 cells. These findings are in agreement with those of Chiang et al (45), who found that miR-192 was downregulated in BGC-803, BGC-823 and SGC-7901 cells.

Finally, we also compared the diagnostic value of miR-122 and miR-192 between GC/DM, GC/NDM and HCs by analyzing the AUC. We found that these two candidate miRNAs could perfectly distinguish GC/DM from both GC/ NDM and the HCs. Furthermore, through Kaplan-Meier survival analysis and Cox analysis, we found that high expression of plasma miR-122 was correlated with prolonged OS of patients with GC, and thus, plasma miR-122 upregulation may be an independent predictor of a more favorable prognosis in patients with GC.

Collectively, our data showed that differential plasma levels of miR-122 and miR-192 exist between patients with GC/DM and patients with GC/NDM. Lower levels of miR-122 and higher levels of miR-192 in plasma were associated with the development of DM in patients with GC. miR-122 and miR-192 may be valuable plasma biomarkers for discerning DM in patients with GC. Therefore, further studies concerning 
the value of detecting plasma miR-122 and miR-192 in the diagnosis of DM in GC in a larger population of patients with $\mathrm{GC}$ and the potential molecular mechanisms by which these miRNAs regulate metastasis of GC are warranted.

\section{Acknowledgements}

This research was supported by the National Basic Research Program of China (973 Program, 2011CB935800), and the National Natural Science Foundation (81071640).

\section{References}

1. Jemal A, Siegel R, Xu J and Ward E: Cancer statistics, 2010. CA Cancer J Clin 60: 277-300, 2010.

2. Ferlay J, Shin HR, Bray F, Forman D, Mathers C and Parkin DM: Estimates of worldwide burden of cancer in 2008: GLOBOCAN 2008. Int J Cancer 127: 2893-2917, 2010.

3. Jemal A, Bray F, Center MM, Ferlay J, Ward E and Forman D: Global cancer statistics. CA Cancer J Clin 61: 69-90, 2011.

4. Yang L: Incidence and mortality of gastric cancer in China. World J Gastroenterol 12: 17-20, 2006.

5. Schlansky B and Sonnenberg A: Epidemiology of noncardia gastric adenocarcinoma in the United States. Am J Gastroenterol 106: 1978-1985, 2011.

6. Kamangar F, Dores GM and Anderson WF: Patterns of cancer incidence, mortality, and prevalence across five continents: defining priorities to reduce cancer disparities in different geographic regions of the world. J Clin Oncol 24: 2137-2150, 2006.

7. Glimelius B, Ekström K, Hoffman K, et al: Randomized comparison between chemotherapy plus best supportive care with best supportive care in advanced gastric cancer. Ann Oncol 8: 163-168, 1997.

8. Murad AM, Santiago FF, Petroianu A, Rocha PR, Rodrigues MA and Rausch M: Modified therapy with 5-fluorouracil, doxorubicin, and methotrexate in advanced gastric cancer. Cancer 72 37-41, 1993.

9. Pyrhonen S, Kuitunen T, Nyandoto P and Kouri M: Randomised comparison of fluorouracil, epidoxorubicin and methotrexate (FEMTX) plus supportive care with supportive care alone in patients with non-resectable gastric cancer. Br J Cancer 71 587-591, 1995

10. Van Cutsem E, Moiseyenko VM, Tjulandin S, et al: Phase III study of docetaxel and cisplatin plus fluorouracil compared with cisplatin and fluorouracil as first-line therapy for advanced gastric cancer: a report of the V325 Study Group. J Clin Oncol 24: 4991-4997, 2006.

11. Roth AD, Fazio N, Stupp R, et al: Docetaxel, cisplatin, and fluorouracil; docetaxel and cisplatin; and epirubicin, cisplatin, and fluorouracil as systemic treatment for advanced gastric carcinoma: a randomized phase II trial of the Swiss Group for Clinical Cancer Research. J Clin Oncol 25: 3217-3223, 2007.

12. Chang YR, Han DS, Kong SH, et al: The value of palliative gastrectomy in gastric cancer with distant metastasis. Ann Surg Oncol 19: 1231-1239, 2012.

13. Li C, Yan M and Zhu ZG: Nonpalliative surgical resection for gastric cancer patients with distant metastasis. J Invest Surg 25 100-106, 2012.

14. Bartel DP: MicroRNAs: genomics, biogenesis, mechanism, and function. Cell 116: 281-297, 2004.

15. Calin GA and Croce CM: MicroRNA signatures in human cancers. Nat Rev Cancer 6: 857-866, 2006.

16. Ambros V: The functions of animal microRNAs. Nature 431: 350-355, 2004

17. Garzon R, Calin GA and Croce CM: MicroRNAs in cancer Annu Rev Med 60: 167-179, 2009.

18. Ortholan C, Puissegur MP, Ilie M, Barbry P, Mari B and Hofman P: MicroRNAs and lung cancer: new oncogenes and tumor suppressors, new prognostic factors and potential therapeutic targets. Curr Med Chem 16: 1047-1061, 2009.

19. Shen J, Stass SA and Jiang F: MicroRNAs as potential biomarkers in human solid tumors. Cancer Lett 329: 125-136, 2012.

20. Allegra A, Alonci A, Campo S, et al: Circulating microRNAs: new biomarkers in diagnosis, prognosis and treatment of cancer (Review). Int J Oncol 41: 1897-1912, 2012.
21. Blanco-Calvo M, Calvo L, Figueroa A, Haz-Conde M, AntónAparicio L and Valladares-Ayerbes M: Circulating microRNAs: molecular microsensors in gastrointestinal cancer. Sensors 12: 9349-9362, 2012.

22. Song MY, Pan KF, Su HJ, et al: Identification of serum microRNAs as novel non-invasive biomarkers for early detection of gastric cancer. PLoS One 7: e33608, 2012.

23. Wang $\mathrm{M}, \mathrm{Gu} \mathrm{H}$, Wang S, et al: Circulating miR-17-5p and miR-20a: molecular markers for gastric cancer. Mol Med Rep 5: 1514-1520, 2012

24. Chen T, Yang K, Yu J, et al: Identification and expansion of cancer stem cells in tumor tissues and peripheral blood derived from gastric adenocarcinoma patients. Cell Res 22: 248-258, 2012.

25. Liu R, Zhang C, Hu Z, et al: A five-microRNA signature identified from genome-wide serum microRNA expression profiling serves as a fingerprint for gastric cancer diagnosis. Eur J Cancer 47: 784-791, 2011.

26. Hu Z, Dong J, Wang LE, et al: Serum microRNA profiling and breast cancer risk: the use of miR-484/191 as endogenous controls. Carcinogenesis 33: 828-834, 2012.

27. Takei Y, Takigahira M, Mihara K, Tarumi Y and Yanagihara K: The metastasis-associated microRNA miR-516a-3p is a novel therapeutic target for inhibiting peritoneal dissemination of human scirrhous gastric cancer. Cancer Res 71: 1442-1453, 2011.

28. Gui J, Tian Y, Wen X, et al: Serum microRNA characterization identifies miR-885-5p as a potential marker for detecting liver pathologies. Clin Sci 120: 183-193, 2011.

29. Hurst DR, Edmonds MD and Welch DR: Metastamir: the field of metastasis-regulatory microRNA is spreading. Cancer Res 69: 7495-7498, 2009

30. Hur K, Toiyama Y, Takahashi M, et al: MicroRNA-200c modulates epithelial-to-mesenchymal transition (EMT) in human colorectal cancer metastasis. Gut 62: 1315-1326, 2012.

31. Valastyan S, Reinhardt F, Benaich N, et al: A pleiotropically acting microRNA, miR-31, inhibits breast cancer metastasis. Cell 137: 1032-1046, 2009.

32. Jopling C, Norman K and Sarnow P: Positive and negative modulation of viral and cellular mRNAs by liver-specific microRNA miR-122. In: Cold Spring Harbor Symposia on Quantitative Biology. Cold Spring Harbor Laboratory Press, pp369-376, 2006.

33. Etiemble J, Möröy T, Jacquemin E, Tiollais P and Buendia M: Fused transcripts of c-myc and a new cellular locus, hcr in a primary liver tumor. Oncogene 4: 51-57, 1989.

34. Girard M, Jacquemin E, Munnich A, Lyonnet S and HenrionCaude A: miR-122, a paradigm for the role of microRNAs in the liver. J Hepatol 48: 648-656, 2008.

35. Wu X, Somlo G, Yu Y, et al: De novo sequencing of circulating miRNAs identifies novel markers predicting clinical outcome of locally advanced breast cancer. J Transl Med 10: 42, 2012.

36. Iino I, Kikuchi H, Miyazaki S, et al: Effect of miR-122 and its target gene cationic amino acid transporter 1 on colorectal liver metastasis. Cancer Sci 104: 624-630, 2013.

37. Tsai WC, Hsu PWC, Lai TC, et al: MicroRNA-122, a tumor suppressor microRNA that regulates intrahepatic metastasis of hepatocellular carcinoma. Hepatology 49: 1571-1582, 2009.

38. Hsu SH, Wang B, Kota J, et al: Essential metabolic, anti-inflammatory, and anti-tumorigenic functions of miR-122 in liver. J Clin Invest 122: 2871-2883, 2012.

39. Gu J, Wang Y and Wu X: MicroRNA in the pathogenesis and prognosis of esophageal cancer. Curr Pharm Des 19: 1292-1300, 2013.

40. Zhao C, Zhang J, Zhang S, et al: Diagnostic and biological significance of microRNA-192 in pancreatic ductal adenocarcinoma. Oncol Rep 30: 276-284, 2013.

41. Schee K, Lorenz S, Worren MM, et al: Deep sequencing the MicroRNA transcriptome in colorectal cancer. PLoS One 8: e66165, 2013.

42. Chiang Y, Song Y, Wang Z, et al: microRNA-192, -194 and -215 are frequently downregulated in colorectal cancer. Exp Ther Med 3: 560-566, 2012

43. Wang G, Chan ES, Kwan BC, et al: Expression of microRNAs in the urine of patients with bladder cancer. Clin Genitourin Cancer 10: 106-113, 2012

44. Kim T, Veronese A, Pichiorri F, et al: p53 regulates epithelialmesenchymal transition through microRNAs targeting ZEB1 and ZEB2. J Exp Med 208: 875-883, 2011.

45. Chiang Y,Zhou X, Wang Z, et al: Expression levels of microRNA192 and -215 in gastric carcinoma. Pathol Oncol Res 18: 585-591, 2012. 